Pacific Journal of Mathematics

INFINITE SUBRINGS OF INFINITE RINGS AND NEAR-RINGS 


\title{
INFINITE SUBRINGS OF INFINITE RINGS AND NEAR-RINGS
}

\author{
HowARD E. BeLL
}

Leffey has proved that every infinite associative ring contains an infinite commutative subring, and thereby suggested the problem of finding reasonably small classes $\mathscr{I}$ of infinite rings with the property that $(*)$ every infinite ring contains a subring belonging to $\mathscr{F}$. Clearly, there is no minimal class $\mathscr{I}$ in the obvious sense, for in any class satisfying $(*)$ a ring may be replaced by any proper infinite subring of itself. In $\S \S 1-3$ we determine a class $\mathscr{J}_{0}$ satisfying $(*)$ and consisting of familiar and easily-described rings; and $\S 4$ we indicate how our results subsume and extend known finiteness results formulated in terms of subrings and zero divisors.

Section 5 identifies classes which satisfy $(*)$ and are minimal in a certain loose sense, and $\S 6$ extends the major result of the first three sections to distributive nearrings. The ring-theoretic results are proved in the setting of alternative rings.

In the remainder of the paper, $Z$ stands for the ring of integers and $Z_{p}$ for the ring of integers modulo $p$, where $p$ is prime. The term $J$-ring refers to a ring $R$ such that for each $x \in R$, there exists an integer $n(x)>1$ for which $x^{n(x)}=x$. The cyclic group of order $p$ is denoted by $C_{p}$, the infinite cyclic group by $C_{\infty}$, the Prüfer $p$-group by $C\left(p^{\infty}\right)$.

Let $p$ be a prime and $\lambda=\left\langle p_{i}\right\rangle$ an infinite strictly-increasing sequence of primes. Then $G(\lambda)$ will denote the direct sum of the groups $C_{p_{i}}, H(\lambda)$ will denote the direct sum of the fields $Z_{p_{i}}$, and $F(p, \lambda)$ will denote the field $\bigcup_{n=1}^{\infty} G F\left(p^{\pi_{n}}\right)$, where $\pi_{n}=p_{1} p_{2} \cdots p_{n}$. If $\lambda$ is replaced by an infinite sequence all terms of which are equal to the same prime $q$, then the analogous groups and rings will be denoted by $G(q), H(q)$, and $F(p, q)$.

Finally, for any subset $S$ of $R, A_{L}(S), A_{R}(S)$, and $A(S)$ will denote respectively the left, right, and two-sided annihilators of $S$.

The major theorem of the paper is

THEOREM 1. Let $\mathscr{F}_{0}$ be the class consisting of all rings of the following kinds:

(a) the zero ring on one of the groups $C_{\infty}, C\left(p^{\infty}\right), G(q)$, or $G(\lambda)$;

(b) rings generated by a single element and isomorphic to a subring of $Z$, to the ring $X Z[X]$, or to the ring $X Z_{p}[X]$ for some prime $p$; 
(c) a ring $H(q)$, a ring $H(\lambda)$, a field $F(p, q)$, or a field $F(p, \lambda)$. Then every infinite alternative ring contains a subring belonging to $\mathscr{F}_{0}$.

1. Preliminary results. We begin this section with some preliminary results on alternative rings. Denoting the associator $(x y) z-x(y z)$ by $(x, y, z)$, we note that it is skew-symmetric and satisfies the identity $[4$, p. 379]

$$
\left(y, x^{2}, z\right)=(y, x, x z+z x)=x(y, x, z)+(y, x, z) x .
$$

LEMma 1. For any alternative ring $R$, the following results hold:

(i) $A(R)$ is a two-sided ideal of $R$;

(ii) if $x \in R, A(x)$ is a subring of $R$;

(iii) if $x \in R$ and $x^{2}=0$, and if $H=A_{R}(x)$, then $H x$ is a zero ring;

(iv) if $e$ is an idempotent of $R$ which commutes elementwise with $R$, then $e$ is in the nucleus and $R$ is the direct sum of the orthogonal ideals $R e$ and $A(e)$.

Proof. (i) The proof is trivial and is omitted.

(ii) Clearly $A(x)$ is an additive subgroup. Also, if $a_{1}, a_{2} \in A(x)$, we have $\left(a_{1}, x, a_{2}\right)=\left(a_{1} x\right) a_{2}-a_{1}\left(x a_{2}\right)=0$; thus $\left(a_{1} a_{2}\right) x=x\left(a_{1} a_{2}\right)=0$.

(iii) Since $H$ is an additive subgroup, so is $H x$. Moreover, letting $h_{1}, h_{2} \in H$ and applying $(A)$, we get $0=\left(h_{1}, 0, h_{2}\right)=\left(h_{1}, x^{2}, h_{2}\right)=$ $\left(h_{1}, x, x h_{2}+h_{2} x\right)=\left(h_{1}, x, h_{2} x\right)=\left(h_{1} x\right)\left(h_{2} x\right)-h_{1}\left(x\left(h_{2} x\right)\right)=\left(h_{1} x\right)\left(h_{2} x\right)$; therefore, $H x$ is a zero ring.

(iv) Taking $x=e$ in $(A)$ and using the skew-symmetry of the associator yields $(e, y, z)=e(e, y, z)+(e, y, z) e=2 e(e, y, z)$. Multiplying through by $e$ then gives $e(e, y, z)=(e, y, z)=0$. The result of (iv) now follows trivially.

Lemma 2. (See [5].) Let $R$ be an infinite alternative ring containing no infinite zero ring. Then for each nilpotent element $x \in R, A(x)$ is infinite.

Proof. Let $G$ denote any infinite additive subgroup of $R$, and define the additive subgroup homomorphism $\phi: G \rightarrow x G$ by $y \mapsto x y$. Application of the first isomorphism theorem shows that either $x G$ is infinite or $\{y \in G \mid x y=0\}=G \cap A_{R}(x)$ is infinite; similarly, one of $G x$ and $G \cap A_{L}(x)$ must be infinite. Using these results, we proceed by induction on the index of nilpotence of $x$.

Suppose first that $x^{2}=0$. Since either $x R$ or $A_{R}(x)$ must be 
infinite, $A_{R}(x)$ is infinite in any event. By (iii) of Lemma $1,\left(A_{R}(x)\right) x$ is a zero ring, hence finite; therefore $A_{R}(x) \cap A_{L}(x)=A(x)$ must be infinite.

Now assume the result for nilpotent elements of index less than $k, k \geqq 3$; and suppose $x^{k}=0$. Since $\left(x^{2}\right)^{k-1}=0, A\left(x^{2}\right)$ is infinite and hence either (i) $x A\left(x^{2}\right)$ is infinite or (ii) $A\left(x^{2}\right) \cap A_{R}(x)$ is infinite. In the event that (i) holds, then one of $\left(x A\left(x^{2}\right)\right) x$ and $x A\left(x^{2}\right) \cap A_{L}(x)$ is infinite; and since both are contained in $A(x)$, we are done. If (ii) holds then either $\left(A\left(x^{2}\right) \cap A_{R}(x)\right) x$ is infinite or $A\left(x^{2}\right) \cap A_{R}(x) \cap A_{L}(x)$ is infinite, and again we are finished because both are contained in $\mathrm{A}(x)$.

Finally, we present for the sake of completeness some easy results on periodic (alternative) rings $R$-that is, rings with the property that for each $x \in R$ there exist distinct positive integers $n, m$ for which $x^{n}=x^{m}$.

LemMA 3. Let $R$ be a periodic alternative ring. Then (i) if $R$ is not nil, $R$ has a non-zero idempotent; (ii) if $R$ has no non-zero nilpotent elements, $R$ is a J-ring.

Proof. (i) If $x^{n}=x^{m}$ for $n>m$, then $x^{j+k(n-m)}=x^{j}$ for each positive integer $k$ and each $j \geqq m$. Thus $x^{m(n-m)}$ is idempotent.

(ii) Let $x^{n}=x^{m}, \quad n>m>1$. Then $x^{m-2} x\left(x-x^{n-m+1}\right)=0=$ $x^{m-2} x^{n-m+1}\left(x-x^{n-m+1}\right)=x^{m-2}\left(x-x^{n-m+1}\right)^{2}$. The obvious induction shows that $x-x^{n-m+1}$ is nilpotent, hence 0 .

2. Initial reduction of the problem.

Proposition 4. Every infinite alternative ring contains an infinite subring of one of the following kinds:

(a) a nil ring;

(b) a ring generated by a single element;

(c) a J-ring.

Proof. Let $R$ be any infinite ring containing no infinite subring of type (a) or (b); note that every infinite subring of $R$ has the same property. Since every element of $R$ generates a finite subring, $R$ must be periodic.

Suppose for the time being that for every set $S_{N}=$ $\left\{0=x_{1}, x_{2}, \cdots, x_{N}\right\}$ of distinct elements of $R$ such that

$$
x_{i} x_{j}=0 \text { for all } i, j=1, \cdots, N
$$

and

$$
R_{N}=A\left(S_{N}\right) \text { is infinite , }
$$


it is possible to find $y \in R_{N} \mid S_{N}$ for which $y^{2}=0$. By (ii) of Lemma 1 and our earlier observations on $R, R_{N}$ is an infinite ring with no infinite zero subring; thus by Lemma 2, the annihilator of $y$ in $R_{N}$, namely $R_{N} \cap A(y)$, must be infinite, and $S_{N+1}=S_{N} \cup\{y\}$ is a set of $N+1$ distinct elements satisfying (4.1) and (4.2). Thus, beginning with $S_{1}=\{0\}$ we can construct inductively an infinite sequence of pairwise-orthogonal elements squaring to zero and therefore an infinite zero ring-a contradiction.

Thus, $R$ contains some set $S_{N}$ satisfying (4.1) and (4.2) such that every element $y$ of $R_{N}$ squaring to zero already belongs to $S_{N}$; replacing $R$ by $R_{N}$, we assume henceforth that $R$ has the property that $S=\left\{x \in R \mid x^{2}=0\right\}$ is finite and equal to $A(R)$.

By (i) of Lemma $3, R$ contains a nonzero idempotent $e$. Now for each $x \in R$, and every nonzero idempotent $e$ of $R$, ex-exe and $x e$ - exe are elements of $R$ squaring to zero, hence are in $S$ and are annihilated by $e$. Thus $x e-e x e=e x-e x e=0$; and by (iv) of Lemma 1 , $e$ is central in $R$ and $R=e R \oplus A(e)$. Since $S \subseteq A(e)$, $e R$ can contain no nonzero elements squaring to zero, hence no nonzero nilpotent elements; thus $e R$ is a $J$-ring. We may assume that $e R$ is finite for all nonzero idempotents $e$, for otherwise we are done. A straightforward induction yields an infinite sequence of pairwise orthogonal nonzero idempotents $e_{i}$ such that for each $m, R=$ $e_{1} R \oplus \cdots \oplus e_{m} R \oplus T_{m}$, where $T_{m}=\bigcap_{i=1}^{m} A\left(e_{2}\right)$. The restricted direct sum $\Sigma \oplus e_{i} R$ of the $J$-rings $e_{2} R$ is therefore an infinite $J$-ring contained in $R$.

Proposition 5. Every infinite alternative nil ring contains an infinite zero ring.

Proof. Assume the result is false. Then by the second and third paragraphs of the proof of Proposition 4, every counterexample must contain as a subring a counterexample $R$ with the property that

(P) $S=\left\{x \in R \mid x^{2}=0\right\}$ is finite and is equal to $A(R)$.

We first show that $R$ must have bounded index of nilpotence. Denote the number of elements of $S$ by $N$, and suppose that $x^{2 k}=0$ for $k \geqq N+1$; note that $x^{k}, \cdots, x^{2 k-1}$ all square to zero. Since $k>N$ these elements cannot be distinct, and there exist positive integers $j_{1}$ and $j_{2}$ such that $j_{1}<j_{2} \leqq 2 k-1$ and $x^{j_{1}}=x^{\left.j_{2}+j_{(j}-j_{1}\right)}$ for all positive integers $j$. Thus $x^{j_{1}}=0$ and it follows that $x^{2 N}=0$ for all $x \in R$.

We assume now that $R$ has degree of nilpotence $K$, minimal for the family of counterexamples with property (P). Clearly $\bar{R}=R / A(R)$ 
is infinite; and since $\left(x^{K-1}\right)^{2}=0$ for all $x \in R, \bar{R}$ must have index of nilpotence at most $K-1$. If $R$ were a counterexample to Proposition 5 , then it would contain a counterexample with property $(\mathrm{P})$, thereby contradicting the minimality of $K$; thus, $\bar{R}$ has an infinite zero subring $\bar{T}=T / A(R)$. Clearly $T$ is an infinite subring of $R$ such that $(x y) z=x(y z)=0$ for all $x, y, z \in T$; in particular, $x^{3}=0$ for all $x \in T$. Since $T$ contains only a finite number of elements squaring to zero, each necessarily of finite additive order, there must exist a positive integer $n$ such that $y^{2}=0$ implies $n y=0$. Thus, $n x^{2}=0$ for all $x \in T$, so that $n T$ has each of its elements squaring to zero, hence is finite. Therefore $\widetilde{T}=\{x \in T \mid n x=0\}$ must be infinite.

Replacing $R$ by $\widetilde{T}$, we now have a counterexample $R$ such that $S=\left\{x \in R \mid x^{2}=0\right\}=A(R)$ is finite, $A(R) \supseteqq R^{2}$, and $n R=0$ for some positive integer $n$. For any finite or infinite sequence $\left\langle x_{i}\right\rangle$ of elements of $R$, denote by $W_{i}$ the subring generated by $S \cup\left\{x_{1}, \cdots, x_{i}\right\}$; and note that each $W_{i}$ must be finite. Using Lemma 2 and (ii) of Lemma 1 we can obtain a sequence $\left\langle x_{i}\right\rangle$ of elements of $R$ such that

$$
\begin{aligned}
& S=W_{0} \subset W_{1} \subset \cdots \subset W_{i} \\
& \text { for each } i, \text { the inclusions all being strict; } \\
& \qquad x_{i} x_{j}=0 \text { for all } i \neq j ; \\
& \text { for each } k, T_{k}=A\left(W_{k}\right) \text { is infinite. }
\end{aligned}
$$

Specifically we begin by choosing any $x_{1} \notin S$ and proceed inductively once $x_{1}, \cdots, x_{k}$ have been defined, the finiteness of $W_{k}$ permits the choice of $x_{k+1} \in T_{k} \backslash W_{k}$; and Lemma 2 applied to $T_{k}$ guarantees that $T_{k} \cap A\left(x_{k+1}\right)=T_{k+1}$ is infinite.

Since $x_{i}^{2} \in S$ for each $i$, the finiteness of $S$ implies the existence of $s \in S$ for which $x_{i}^{2}=s$ for $n$ distinct $x_{i}$. Letting $z$ be the sum of these $x_{i}$, we have the result that $z^{2}=n s=0$ but $z \notin S$. This contradiction completes the proof of Proposition 5.

Proposition 6. Every infinite alternative ring contains an infinite subring which is both associative and commutative.

Proof. Since one-generator subrings and zero rings are obviously associative and commutative, we need only establish the same for alternative $J$-rings. These are commutative by a theorem of Smiley [10]; the associativity follows from the general result that a commutative alternative ring with no nonzero nilpotent elements is associative [7, Lemma 3].

3. Proof of Theorem 1. The proof of Theorem 1 is completed 
by three lemmas, which further refine the classes (a), (b) and (c) of Proposition 4 (in that order). In view of Proposition 6, we may assume that our rings are associative.

LEMMA 7. Every infinite zero ring contains a zero ring on one of the following groups: (i) $C_{\infty}$; (ii) $G(\lambda)$ for some strictly-increasing sequence $\lambda$ of primes; (iii) $G(q)$ for some prime $q$; (iv) $C\left(p^{\infty}\right)$ for some prime p.

Proof. Of course, we wish to prove that every infinite abelian group contains one of the indicated groups as a subgroup.

Suppose then that $G$ is any infinite abelian group. If $G$ contains an element of infinite order, it contains an infinite cyclic subgroup; hence we may suppose that $G$ is periodic, in which case $G=\Sigma \oplus G_{p}$, where the $G_{p}$ are the $p$-primary components for all primes $p$. If there are infinitely many nontrivial $G_{p}$, then $G$ has a subgroup of type (ii); thus we consider the case of only finitely many nontrivial $G_{p}$ and assume without loss that $G$ is a countable p-group for some prime $p$. Let $H$ be the subgroup of $G$ consisting of elements of order $p$.

If $G$ has no elements of infinite height, then $G$ has a subgroup of type (iii) by Theorem 11.3 of [3]; if $H$ is infinite, then we can replace $G$ by $H$ and apply the same argument. Thus, we suppose that $H$ is finite and that $G$ contains an element $x_{0}$ of infinite height such that $p x_{0}=0$. There exists a sequence $x_{i}$ of elements of $G$ for which $p^{i} x_{i}=x_{0}, i=1,2, \cdots$; and the set $\left\{x_{1}-p^{i-1} x_{i} \mid i=2,3, \cdots\right\}$ is a subset of $H$. There is, therefore, a smallest integer $M \geqq 2$ for which $p^{M-1} x_{M}$ is equal to $p^{i-1} x_{i}$ for infinitely many $i$; and it follows that $x_{1}^{\prime}=p^{M-1} x_{M}$ is of infinite height and $p x_{1}^{\prime}=x_{0}$. Proceeding inductively, we get a sequence $x_{0}, x_{1}, x_{2}, \cdots$ where $p x_{0}=0$ and $p x_{i}=x_{i-1}, i=1,2, \cdots$; hence $G$ must contain $C\left(p^{\infty}\right)$ as a subgroup.

LEMMA 8. Let $R$ be an infinite ring which is generated by a single element, and suppose $R$ contains no infinite zero ring. Then $R$ must contain $X Z[X]$, or $X Z_{p}[X]$ for some prime $p$, or a subring of $Z$.

Proof. Suppose initially that $R$ is generated by an element $a$ of infinite additive order. Clearly, if $a$ is not algebraic over the integers, $R \cong X Z[X]$. Consider now the case where $a$ is algebraic over the integers, and let $a$ satisfy

$$
n_{1} a^{k_{1}}+n_{2} a^{k_{2}}+\cdots+n_{s} a^{k_{s}}=0,
$$

where $k_{1}<k_{2}<\cdots<k_{s}, \quad n_{1} \neq 0$, and $k_{1}$ is the smallest positive 
integer occurring as the lowest power of $a$ in any such relation. If $k_{1}>1$, then $a b=0$, where $b=n_{1} a^{k_{1}-1}+\cdots+n_{s} a^{k_{s}-1}$; and since the annihilator of $a$ is the annihilator of $R$, which under our assumptions is finite, either $b=0$ or $j b=0$ for some positive integer $j$. In either case the minimality of $k_{1}$ is contradicted; therefore $k_{1}=1$ and

$$
n_{1} a=a p(a),
$$

where $p(X) \in Z[X]$ has zero constant term. Letting $b=p(a)$ in (8.2), we see that $b$ has infinite order and $b^{2}=n_{1} b$. Thus, the subring of $R$ generated by $b$ is isomorphic to the subring of $Z$ generated by $n_{1}$.

We turn now to the case where the generator $a$ has finite order $n=p_{1}^{\alpha_{1}} p_{2}^{\alpha_{2}} \cdots p_{k}^{\alpha_{k}}$, the $p_{i}$ being distinct primes. Since $R$ is the direct sum of its $p_{i}$-primary components and since each of these is generated by a single element, we may assume that $n=p^{a}$ for some prime $p$. If $\alpha=1$, in which case $R$ may be regarded as an algebra over $Z_{p}$, then $R \cong X Z_{p}[X]$; otherwise the generator would be algebraic over $Z_{p}$ and hence $R$ would be finite.

Suppose, then, that $\alpha>1$. Since $p R$ is nil, it must be finite by Proposition 5; therefore $\widetilde{R}=\{x \in R \mid p x=0\}$ is infinite. If $a$ denotes the generator of $R$, then for appropriate positive integers $n, m$ we have $p a^{n}=p a^{m}=p a^{m+k(n-m)}$ for all integers $k \geqq 1$; and it follows that $b=\sum_{j=1}^{p^{\alpha-1}} a^{m+j(n-m)}$ is an element of $\widetilde{R}$. Moreover, $b \neq 0$ since $a$ would otherwise generate a finite ring. Clearly $b$ cannot be algebraic over $Z_{p}$-that too would imply $R$ is finite; hence $b$ generates a subring isomorphic to $X Z_{p}[X]$.

Lemma 9. Let $R$ be an infinite J-ring. Then $R$ must contain a subring of one of the following forms: $H(\lambda)$ for some strictlyincreasing sequence $\lambda$ of primes; $H(q)$ for some prime $q$; a field $F(p, \lambda)$ for some strictly-increasing sequence of primes; a field $F(p, q)$.

Proof. Let $R$ be any infinite $J$-ring. Since the additive group of a $J$-ring is a torsion group, $R=\Sigma \oplus R_{p}$, where $R_{p}$ are the $p$-primary components of $R^{+}$. Clearly each nontrivial $R_{p}$ contains a non-zero idempotent of additive order $p$, hence $R$ contains a ring $H(\lambda)$ if there are infinitely may nontrivial $R_{p}$. Otherwise we may assume that the additive group of $R$ is a $p$-group; moreover, since $R$ has no nonzero nilpotent elements, the additive order of each nonzero element is square-free and we have $p R=0$. If $x \in R$ satisfies $x^{n+1}=x$, then $x^{n}=e$ is an idempotent such that $e x=x e=x$; thus if $R$ has a unique nonzero idempotent, it is an identity element 
and $R$ is a field. On the other hand, if $e$ is a nonzero idempotent which is not an identity element, we get a nontrivial decomposition $R=R e \oplus A(e)$ with one of the summands infinite. Thus, by continuing direct sum decompositions as long as possible, we either get a ring $H(p)$ or an infinite field. The proof is completed by showing that every infinite $J$-field contains a subfield of type $F(p, \lambda)$ or $F(p, q)$.

Accordingly, let $F$ be an infinite $J$-field and note that every finitely-generated subring is a finite field. Thus, $F$ contains a subfield $\widetilde{F}$ which is the union of a strictly ascending tower $Z_{p}=F_{0} \subset F_{1} \subset F_{2} \ldots$ of finite fields; and we may assume that the tower has been so refined that there are no subfields properly contained between any two of its members. It follows that for each $i=1,2, \cdots,\left[F_{i}: F_{i-1}\right]$ is a prime $p_{i}$. Using the basic facts about finite fields, it is easy to construct a field $F(p, \lambda)$ if there are infinitely many different $p_{i}$ and a field $F(p, q)$ otherwise. This completes the proof of Lemma 9 and hence of Theorem 1 .

4. Some consequences of Theorem 1. Theorem 1 leads directly to the following two extensions of Szele's result [11] that an associative ring must be finite if it has both a.c.c. and d.c.c. on subrings.

THEOREM 2. If $R$ is an alternative ring satisfying both ascending chain condition and descending chain condition on commutative associative subrings, then $R$ is finite.

Proof. If there were a counterexample the class $\mathscr{F}_{0}$ of Theorem 1 would include rings having both a.c.c. and d.c.c. on subrings; but it does not.

Theorem 3. Let $R$ be an alternative ring having only a finite number of zero subrings and a finite number of subrings which are integral domains. Then $R$ is finite.

Proof. The rings of type (a) in Theorem 1 all contain infinitely many zero subrings; those of types (b) and (c) all contain infinitely many integral domains.

REMARKS 1. Of course we could have obtained Theorem 2 by invoking our Proposition 6 and Szele's proof for the associative case. The proof of Theorem 1, however, is conceptually more elementary than Szele's proof.

2. In the hypotheses of Theorem 3, finiteness cannot be replaced 
by a.c.c. and d.c.c., as can be seen by considering the ring $H(\lambda)$ for a strictly-increasing sequence $\lambda$.

The following theorem, which presents a new finiteness criterion for rings, does not seem to be a direct corollary of Theorem 1 but uses some of the machinery from its proof.

THEOREM 4. Let $R$ be an alternative ring having nonzero divisors of zero. If $A(x)$ is finite for each nonzero (two-sided) zero divisor $x$, then $R$ is finite.

Proof. Suppose that $R$ is an infinite ring with nonzero divisors of zero. If $R$ has nonzero nilpotent elements, then by Lemma 2 , $R$ has a nonzero element $x$ for which $A(x)$ is infinite; thus, assume that $R$ has no nonzero nilpotent elements, in which case $a b=0$ if and only if $b a=0$, so that there is no distinction between right and left annihilators. If for some nonzero pair $a, b$ we have $a b=0$ and $a$ generating an infinite subring, then $A(b)$ is infinite; if there exists no such pair, for each nonzero zero divisor $a$, we have $a^{m}=a^{n}$ for distinct positive integers $n, m$ and some power of $a$ is a nonzero idempotent, necessarily central. In the latter case, the decomposition $R=R e \oplus A(e)$ is nontrivial with at least one of the summands infinite, so we again have a nonzero $x$ with $A(x)$ infinite.

An immediate consequence of Theorem 4 is the following theorem which extends Theorem 3 of [1].

THEOREM 5. If $R$ is an alternative ring with nonzero divisors of zero and has a.c.c. and d.c.c. on subrings consisting of twosided zero divisors of $R$, then $R$ is finite.

Our final application of Theorem 1 deals with the question of when an infinite ring contains infinitely many infinite subrings.

THEOREM 6. If $R$ is an infinite alternative ring containing no zero ring on a Prüfer p-group and no field $F(p, q)$, then $R$ has infinitely many infinite (commutative associative) subrings. In particular, if $R$ contains no infinite subring whose subrings are totally ordered by inclusion, $R$ must have infinitely many infinite subrings.

Proof. The first assertion is obtained by noting that all the members of the class $\mathscr{J}_{0}$ with the exception of zero rings on groups $C\left(p^{\infty}\right)$ and fields $F(p, q)$ contain infinite decreasing sequences of infinite rings. The second assertion is immediate from the fact that 
the zero rings on the groups $C\left(p^{\infty}\right)$ and the fields $F(p, q)$ are precisely the infinite rings whose subrings are totally ordered by inclusion (see [9]).

5. Minimality considerations. In this section, we deal with a notion of minimality for classes satisfying (*).

If $\mathscr{F}$ is any such class of rings, it must obviously contain all infinite rings having no proper infinite subrings-specifically, all fields $\mathrm{F}(p, q)$ and the zero ring on each group $C\left(p^{\infty}\right)$; and it must contain all infinite rings which are isomorphic to each of their proper infinite subrings-i.e., the zero ring on $C_{\infty}$, the zero ring on each group $G(p)$, and all rings $H(p)$. It must include at least one decreasing sequence of subrings of $Z$, at least one subring of $X Z[X]$, and at least one subring of $X Z_{p}[X]$ for each prime $p$. Finally, it must include infinitely many rings of the form $H(\lambda)$, infinitely many zero rings on groups $G(\lambda)$, and infinitely many fields $F(p, \lambda)$; this fact follows at once from the observation that infinite subrings of rings of these types are of the same type.

Such a class $\mathscr{J}$ need not contain more than one decreasing sequence of rings $n_{i} Z$ provided that the one sequence has the property that each nonzero integer $n$ divides some $n_{i}$; and since every subring of $X Z[X]$ or $X Z_{p}[X]$ contains a subring isomorphic to the entire ring, it will be sufficient for $\mathscr{J}$ to contain any one subring of $X Z] X]$ and any one subring of each ring $X Z_{p}[X]$.

It is not clear exactly which classes of rings $H(\lambda), F(p, \lambda)$, and zero rings on $G(\lambda)$ must be included in $\mathscr{F}$, but we can say something. Let $\lambda_{0}$ denote the sequence of all primes of $Z$ in their natural order, and let $J$ denote any strictly-increasing sequence of positive integers. Denote by $\lambda_{J}$ the subsequence of $\lambda_{0}$ obtained by choosing those terms indexed by $J$. Then $\mathscr{F}$ need contain no rings $H\left(\lambda_{J}\right)$ where $J$ has bounded gaps; similar considerations apply to fields $F(p, \lambda)$ and zero rings on $G(\lambda)$.

A set $\mathscr{J}$ of strictly-increasing sequences of positive integers will be called adequate if each of its members has an unbounded set of gap lengths and if it contains at least one subsequence of every strictly-increasing sequence of positive integers. It being understood that $\mathcal{J}$ in each occurrence denotes an adequate set of sequences, we now define a class $\mathscr{J}$ satisfying $\left(^{*}\right)$ to be irredundant if it includes each of the following:

(i) all fields $F(p, q)$ and the zero ring on each group $C\left(p^{\infty}\right)$;

(ii) the zero ring on $C_{\infty}$, the zero ring on each group $G(p)$, and the ring $H(p)$ for each prime $p$;

(iii) one infinite decreasing sequence $\left\langle n_{i} Z\right\rangle$ of subrings of $Z$, with the property that each positive integer $n$ divides some $n_{i}$; 
(iv) one nonzero subring of $X Z[X]$; and one nonzero subring of $X Z_{p}[X]$ for each prime $p$;

(v) the zero rings on any family of groups of the form $\left\{G\left(\lambda_{J}\right) \mid J \in \mathscr{J}\right\}$

(vi) any family $\left\{H\left(\lambda_{J}\right) \mid J \in \mathscr{J}\right\}$;

(vii) for each prime $p$, one family $\left\{F\left(p, \lambda_{J}\right) \mid J \in \mathscr{J}\right\}$.

We can now state a characterization theorem. A formal proof is omitted, since most of the details are included in the preceding discussion.

TheOREM 7. A class $\mathscr{J}$ of rings satisfies $\left(^{*}\right)$ if and only if it contains an irredundant subclass.

It would, of course, be interesting to know more about adequate sets of sequences; but we are unable to give a tight description of them. It is clear, however, that they are quite large-in fact, it is easily shown that an adequate set contains uncountably many subsequences of each increasing sequence of positive integers.

6. Extensions to distributive near-rings, A near-ring $R$ is a binary system satisfying all the (associative) ring axioms except right distributivity and commutativity of addition; $R$ is called a distributive near-ring (dnr) if it does have right distributivity. An ideal of a $d n r R$ is a normal subgroup of $R^{+}$which is closed under left and right multiplication by elements of $R$; the theory of homomorphisms is the same as for rings.

A recurring consideration in the study of near-rings is the relationship between distributivity and additive commutativity. By extending our earlier results a bit, we can show that "most" infinite distributive near-rings contain infinite sub-near-rings which are additively commutative, hence rings. Clearly, not all dnr's have this property, for there exist infinite groups with no infinite abelian subgroups-we shall refer to them as exceptional [8, p. 35]-and the near-ring with trivial multiplication on such a group has no infinite subrings.

We shall make use of two well-known elementary results on dnr's-

(I) If $R$ is a distributive near-ring, $R^{2}$ is a ring [2].

(II) If $R$ is a distributive near-ring and $R^{\prime}$ is the derived group of the additive group $R^{+}$, then $R^{\prime}$ is an ideal of $R$ and $R R^{\prime}=R^{\prime} R=$ $0[6]$.

THEOREM 8. Let $R$ be an infinite distributive near-ring for which the derived group of $R^{+}$is not exceptional. Then $R$ contains 
an infinite subring.

Proof. In view of (I) and (II) we consider only $R$ with both $R^{2}$ and $R^{\prime}$ finite. The finiteness of $R^{2}$ implies the existence of a positive integer $n$ such that $n x^{2}=0$ for all $x \in R$; thus, if $u$ is an element of $R$ having infinite additive order, we have $(n u)^{2}=0$ and $n u$ generates an infinite ring. Therefore, we may assume henceforth that $R^{+}$is a periodic group. Another consequence of the finiteness of $R^{2}$ is that $R$ is a periodic near-ring-i.e., for each $x \in R$, there are distinct positive integers $n, m$ for which $x^{n}=x^{m}$.

Observe that $R / R^{\prime}$ is an infinite ring. If it has no infinite zero ring, then by Theorem 1 it contains an infinite subring $S / R^{\prime}$ with no nonzero nilpotent elements. Now by Lemma 3 periodic rings with no nonzero nilpotent elements are $J$-rings, hence are commutative by Jacobson's well-known theorem; and it follows that for all $x, y \in S, x y-y x \in R^{\prime} \subseteq A(R)$. In particular, if $e$ is an idempotent of $S$ and $s$ an arbitrary element of $S$, then $(e s-s e) e=e(e s-s e)=0$ and therefore idempotents of $S$ are in the centre of $S$. Since $e \in e S \subseteq R^{2}$, we easily obtain a finite set $E$ of pairwise-orthogonal idempotents such that $S_{0}=S \cap A(E)$ is infinite and contains no nonzero idempotents; and because $S_{0}$ is periodic, it must be nil. Since $S_{0}$ is infinite, we cannot have $S_{0} \subseteq R^{\prime}$; thus, we have contradicted the fact that $S / R^{\prime}$ had no nonzero nilpotent elements.

To complete the proof, we need only discuss the case where $R^{\prime}$ and $R^{2}$ are both finite and $R / R^{\prime}$ contains an infinite zero ring $S / R^{\prime}$. For $x, y \in S$, we must have $x y \in R^{\prime} \subseteq A(R)$, so in particular $x^{3}=0$ for all $x \in S$.

By applying an inductive argument similar to that used in the proof of Proposition 5, we can show that $S$ must contain an infinite sequence $\left\langle x_{i}\right\rangle$ of pairwise-orthogonal elements squaring to zero. We omit the datails, but mention that Lemma 2 holds in the context of dnr's and that the ability to choose $x_{i+1}$ not in the subring generated by $\left\{x_{1}, \cdots, x_{n}\right\}$ depends on local finiteness of $R^{+}$, which is guaranteed by the fact that $R^{+}$is a periodic group with finite derived group.

Consider all additive commutators of the form $\left[x_{1}, x_{i}\right]=x_{1}+x_{i}-$ $x_{1}-x_{i}, i>1$. Since $S^{\prime}$ is finite, we may assume that $\left[x_{1}, x_{i}\right]=$ $\left[x_{1}, x_{j}\right]$ for all $i, j>1$.

Defining the sequence $\left\langle u_{i}\right\rangle$ by $u_{1}=x_{1}$ and $u_{j}=-x_{2}+x_{j+1}$ for $j>1$, we obtain a sequence of pairwise-orthogonal elements squaring to zero, such that no $u_{i}$ belongs to the additive subgroup generated by the previous terms and such that all its terms commute additively with $u_{1}$ Continuing with the inductive construction this suggests, we arrive at a sequence of pairwise orthogonal elements squaring to zero and generating an infinite abelian subgroup of $S^{+}$; 
therefore $S$ contains an infinite zero ring. The proof of Theorem 8 is now complete.

COROLlaRY 1. If $R$ is an infinite distributive near-ring, then $R$ contains an infinite ring or an infinite near-ring with trivial multiplication.

COROLlARY 2. If $R$ is an infinite distributive near-ring having ascending chain condition and descending chain condition on subnear-rings and if $R^{\prime}$ is not exceptional, then $R$ is finite.

\section{Proof. Use Theorem 2 and Theorem 8.}

REMARK. Whether the hypothesis that $R^{\prime}$ is not exceptional is required in Corollary 2 is equivalent to the unsolved problem as to whether a group with acc and dcc on subgroups need be finite.

CoROLlaRY 3. Let $R$ be an infinite distributive near-ring with solvable additive group. Then $R$ contains an infinite subring.

Proof. Let $R^{(i)}$ denote the $i$ th term of the derived series of $R^{+}, i=1,2, \cdots$. Since $R^{+}$is solvable, there is a smallest positive integer $m$ for which $R^{(m)}$ is finite. If $m=1, R^{\prime}$ is not exceptional and we are finished; if $m>1$, then $R^{(m-1)}$ is an infinite distributive near-ring whose derived group is not exceptional, and $R^{(m-1)}$ contains an infinite ring.

COROLLARY 4. If $R$ is an infinite distributive near-ring the additive group of which is locally finite, then $R$ must contain an infinite subring.

Proof. If $R^{\prime}$ is not finite, it must contain an infinite abelian subgroup by the Hall-Kulatilaka-Kargopolov theorem [8, p. 95]; therefore $R^{\prime}$ is not exceptional, and the result follows from Theorem 8.

\section{REFERENCES}

1. H. E. Bell, Rings with finitely many subrings, Math. Ann., 182 (1969), 314-318.

2. A. Fröhlich, Distributively generated near-rings, I, Ideal theory, Proc. London Math. Soc., (3) 8 (1958), 76-94.

3. L. Fuchs, Abelian Groups, Pergamon, Oxford, 1960.

4. M. Hall, The Theory of Groups, Macmillan, New York, 1959.

5. T. J. Laffey, On commutative subrings of infinite rings, Bull. London Math. Soc., 4 (1972), 3-5. 
6. S. Ligh, On commutativity of near-rings, Kyungpook Math. J., 10 (1970), 105106.

7. K. McCrimmon, Finite power-associative division rings, Proc. Amer. Math Soc., 17 (1966), 1173-77.

8. D. J. S. Robinson, Finiteness Conditions and Generalized Soluble Groups, Part 1, Springer-Verlag, 1972.

9. A. Rosenfeld, $A$ note on two special types of rings, Scripta Mathematica, 28 (1967), 51-54.

10. M. Smiley, Alternative regular rings without nilpotent elements, Bull. Amer. Math. Soc., 53 (1947) 775-778.

11. T. Szele, On a finiteness criterion for modules, Publ. Math. Debrecen 3 (1954), 253-256.

Received May 21, 1975.

BROCK UNIVERSITY 


\title{
PACIFIC JOURNAL OF MATHEMATICS
}

\section{EDITORS}

RichaRd ARENS (Managing Editor)

University of California

Los Angeles, California 90024

\section{R. A. Beaumont}

University of Washington

Seattle, Washington 98105
J. DUGUNDJI

Department of Mathematics University of Southern California Los Angeles, California 90007

D. Gilbarg and J. Milgram

Stanford University

Stanford, California 94305

\section{ASSOCIATE EDITORS}
E. F. BECKENBACH
B. H. NeUmanN
F. WOLF
K. YoshIDA

\section{SUPPORTING INSTITUTIONS}

\author{
UNIVERSITY OF BRITISH COLUMBIA \\ CALIFORNIA INSTITUTE OF TECHNOLOGY \\ UNIVERSITY OF CALIFORNIA \\ MONTANA STATE UNIVERSITY \\ UNIVERSITY OF NEVADA \\ NEW MEXICO STATE UNIVERSITY \\ OREGON STATE UNIVERSITY \\ UNIVERSITY OF OREGON \\ OSAKA UNIVERSITY
}

\author{
UNIVERSITY OF SOUTHERN CALIFORNIA \\ STANFORD UNIVERSITY \\ UNIVERSITY OF TOKYO \\ UNIVERSITY OF UTAH \\ WASHINGTON STATE UNIVERSITY \\ UNIVERSITY OF WASHINGTON \\ AMERICAN MATHEMATICAL SOCIETY
}

The Supporting Institutions listed above contribute to the cost of publication of this Journal, but they are not owners or publishers and have no responsibility for its content or policies.

Mathematical papers intended for publication in the Pacific Journal of Mathematics should be in typed form or offset-reproduced, (not dittoed), double spaced with large margins. Underline Greek letters in red, German in green, and script in blue. The first paragraph or two must be capable of being used separately as a synopsis of the entire paper. Items of the bibliography should not be cited there unless absolutely necessary, in which case they must be identified by author and Journal, rather than by item number. Manuscripts, in triplicate, may be sent to any one of the editors. Please classify according to the scheme of Math. Reviews, Index to Vol. 39. All other communications should be addressed to the managing editor, or Elaine Barth, University of California, Los Angeles, California, 90024.

The Pacific Journal of Mathematics expects the author's institution to pay page charges, and reserves the right to delay publication for nonpayment of charges in case of financial emergency.

100 reprints are provided free for each article, only if page charges have been substantially paid. Additional copies may be obtained at cost in multiples of 50 .

The Pacific Journal of Mathematics is issued monthly as of January 1966. Regular subscription rate: $\$ 72.00$ a year (6 Vols., 12 issues). Special rate: $\$ 36.00$ a year to individual members of supporting institutions.

Subscriptions, orders for back numbers, and changes of address should be sent to Pacific Journal of Mathematics, 103 Highland Boulevard, Berkeley, California, 94708.

PUBLISHED BY PACIFIC JOURNAL OF MATHEMATICS, A NON-PROFIT CORPORATION

Printed at Kokusai Bunken Insatsusha (International Academic Printing Co., Ltd.), 8-8, 3-chome, Takadanobaba, Shinjuku-ku, Tokyo 160, Japan.

Copyright (C) 1975 by Pacific Journal of Mathematics Manufactured and first issued in Japan 


\section{Pacific Journal of Mathematics}

\section{Vol. 59, No. $2 \quad$ June, 1975}

Aharon Atzmon, A moment problem for positive measures on the unit disc ........

Peter W. Bates and Grant Bernard Gustafson, Green's function inequalities for

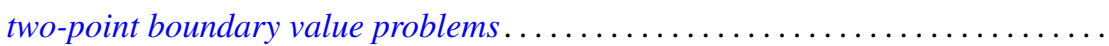

Howard Edwin Bell, Infinite subrings of infinite rings and near-rings ...........

Grahame Bennett, Victor Wayne Goodman and Charles Michael Newman, Norms of

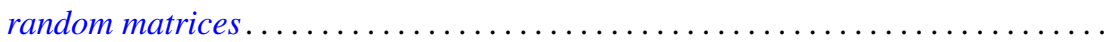

Beverly L. Brechner, Almost periodic homeomorphisms of $E^{2}$ are periodic.........

Beverly L. Brechner and R. Daniel Mauldin, Homeomorphisms of the plane ........

Jia-Arng Chao, Lusin area functions on local fields ......................

Frank Rimi DeMeyer, The Brauer group of polynomial rings ...............

M. V. Deshpande, Collectively compact sets and the ergodic theory of

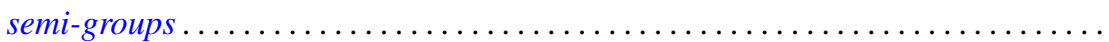

Raymond Frank Dickman and Jack Ray Porter, $\theta$-closed subsets of Hausdorff

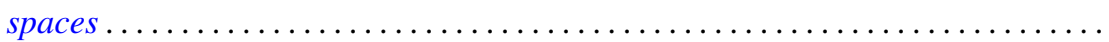

Charles P. Downey, Classification of singular integrals over a local field .......... 407

Daniel Reuven Farkas, Miscellany on Bieberbach group algebras . . . . . . . . . . . .

Peter A. Fowler, Infimum and domination principles in vector lattices . . . . . . . . . .

Barry J. Gardner, Some aspects of T-nilpotence. II: Lifting properties over

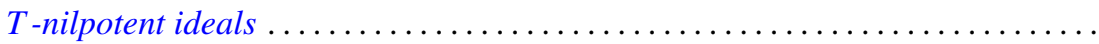

Gary Fred Gruenhage and Phillip Lee Zenor, Metrization of spaces with countable

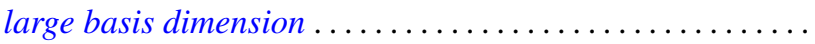

J. L. Hickman, Reducing series of ordinals...

Hugh M. Hilden, Generators for two groups related to the braid group ...

Tom (Roy Thomas Jr.) Jacob, Some matrix transformations on analytic sequence

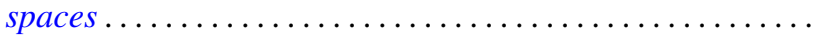

Elyahu Katz, Free products in the category of $k_{w}$-groups . .....

Tsang Hai Kuo, On conjugate Banach spaces with the Radon-Nikodým property...

Norman Eugene Liden, $K$-spaces, their antispaces and related mappings ...

Clinton M. Petty, Radon partitions in real linear spaces ........

Alan Saleski, A conditional entropy for the space of pseudo-Menger maps ....

Michael Singer, Elementary solutions of differential equations .

Eugene Spiegel and Allan Trojan, On semi-simple group algebras. I. . .

Charles Madison Stanton, Bounded analytic functions on a class of open Riemann

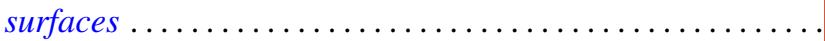

Sherman K. Stein, Transversals of Latin squares and their generalizations ....

Ivan Ernest Stux, Distribution of squarefree integers in non-linear sequences . . .

Lowell G. Sweet, On homogeneous algebras ................

Lowell G. Sweet, On doubly homogeneous algebras .......... .

Florian Vasilescu, The closed range modulus of operators ......

Arthur Anthony Yanushka, A characterization of the symplectic groups $\operatorname{PSp}(2 m, q)$

as rank 3 permutation groups... 